\title{
Aberrant ketolysis fuels hepatocellular cancer progression
}

\author{
Cell Research (2016) 26:1077-1078. doi:10.1038/cr.2016.110; published online 20 September 2016
}

At odds with their normal counterparts, hepatocellular carcinoma cells efficiently utilize ketone bodies to proliferate despite serum deprivation. These findings, which have been recently published in Cell Research, identify a novel metabolic circuitry through which tumors successfully cope with adverse microenvironmental conditions.

One of the most impressive features of malignant cells is their ability to adapt to prominent changes in the composition of the extracellular milieu [1]. At odds with their non-transformed counterparts, cancer cells are indeed able to proliferate in the absence of growth factors, under pronounced hypoxia, as well as when nutrients and amino acids are limited [1]. Throughout the past decade, work from several laboratories clarified that neoplastic cells facing adverse microenvironmental conditions can utilize a variety of metabolites to support catabolic and anabolic reactions, including (but presumably not limited to) glucose, acetate, lactate, creatine, glutamine, serine, glycine and fatty acids [2]. Thus, cancer cells generally rewire their metabolism, hence acquiring the capacity to utilize metabolites that are locally available to support tumor progression [2]. Although less universal and less specific than initially thought (meaning that different cancers can exhibit quite distinct metabolic shifts, and that at least some of the metabolic alterations that accompany malignancy are also found in highly proliferating non-transformed cells), such a rewiring process provides putative targets for the development of novel anticancer agents [3]. Recent work from Huafeng Zhang's group identifies a novel metabolic circuitry based on ketolysis through which hepatocellular carcinoma (HCC) cells proliferate in spite of adverse microenvironmental conditions [4].

Starting from the observation that HCC cells downregulate fatty acid oxidation in response to hypoxia as a mechanism to relieve oxidative stress and support proliferation [5], Zhang and collaborators initially set out to investigate the expression levels of genes involved in lipid metabolism in human HCC HepG2 cells deprived of glucose, glutamine or serum by quantitative real-time PCR. In response to all these microenvironmental challenges, HepG2 cells upregulated multiple enzymes involved in ketogenesis [6], including BDH1, HMGCL, HMGCS1 and HMGCS2, consistent with the prominent role played by the liver in the provision of ketone bodies to other tissues in response to starvation. Surprisingly, HepG2 cells subjected to serum (but not glucose or glutamine) deprivation also exhibited increased levels of 3-oxoacid CoA-transferase 1 (OXCT1), which catalyzes the rate-limiting reaction of ketolysis and is normally not expressed in the adult liver [7]. Similar results were obtained with other human HCC cell lines, including Hep3B and PLC cells, and OXCT1 upregulation driven by serum withdrawal was confirmed by immunoblotting [4]. In line with this finding, exogenously supplied $\beta$-hydroxybutyrate (a ketone body) was converted by HCC cells subjected to starvation (but not by non-transformed human liver THLE3 cells, nor by HCC cells maintained in control conditions) into various intermediates of the tricyclic acid cycle, including citrate, succinate, fumarate, malate, glutamate and aspartate, supporting a time-dependent recovery in intracellular ATP levels and proliferation rates [4]. Importantly, the stable downregulation of OXCT1 by two distinct shRNA-coding constructs virtually abolished the ability of HepG2 cells undergoing serum deprivation to catabolize exogenous $\beta$-hydroxybutyrate. Moreover, OXCT1-depleted HepG2 cells exhibited exacerbated ATP loss in response to serum deprivation as compared to HepG2 cells expressing a control shRNA, and were completely unable to proliferate. Such defects could be rescued by the transgene-driven overexpression of a non-interferable OXCT1 variant. Of note, $\beta$-hydroxybutyrate was not exogenously supplied in this last set of experiments, suggesting that $\mathrm{HCC}$ can employ endogenously produced ketone bodies to proliferate despite serum deprivation. Corroborating this hypothesis, the depletion of HMGCS2 virtually abolished the proliferative advantage conferred to serum-depleted HepG2 cells by OXCT1 overexpression, unless exogenous $\beta$-hydroxybutyrate was added to the culture medium [4].

Next, Zhang and colleagues investigated the signal transduction pathways responsible for the upregulation of OXCT1 in serum-deprived HCC cells by interrogating a panel of chemical inhibitors. Amongst a few molecules with distinct specificities, LY-294002 (an inhibitor of phosphoinositide-3-kinases and AKT1) was the sole agent capable of avoiding OXCT1 upregulation by serum withdrawal. Corroborating a direct implication of AKT1 in this process, HepG2 cells expressing two distinct AKT1-specific shRNAs lost their ability to upregulate OXCT1 upon serum deprivation, as did HepG2 cells depleted of an essential component of the AKT1 
activator mechanistic target of rapamycin (MTOR) complex 2 (mTORC2), namely, RPTOR independent companion of MTOR, complex 2 (RICTOR). These findings were further confirmed with rapamycin, a macrolide that activates mTORC2 as it inhibits mTORC1 [8]. Moreover, the ability of mTORC2-activated AKT1 to drive the upregulation of OXCT1 was mechanistically ascribed to the transcription factor SP1. Thus, similar to their AKT1-deprived counterparts, SP1-depleted HepG2 cells were unable to catabolize exogenous $\beta$-hydroxybutyrate [4]. Interestingly, the ability of HepG2 cells to upregulate OXCT1 was associated with limited activation of the energy sensor AMP-activated protein kinase (AMPK) upon serum withdrawal [9], presumably reflecting an improved capacity to preserve bioenergetic homeostasis via ketolysis. Accordingly, depletion of OXCT1 or AKT1 aggravated, while OXCT1 overexpression quenched, the autophagic response of HepG2 cells to serum deprivation, knowing that autophagy is an evolutionarily ancient mechanism of adaptation to adverse microenvironmental conditions [10,11]. Exogenous $\beta$-hydroxybutyrate also limited autophagy in serum-starved HepG2 cells, provided that OXCT1 was normally expressed. Moreover, the overexpression of OXCT1 abolished the ability of an AKT1-specific shRNA to exacerbate serum starvation-induced autophagy [4].

Finally, Zhang and collaborators validated the relevance of their findings in vivo. OXCT1-overexpressing HepG2 cells xenografted into athymic $n u / n u$ mice manifested increased proliferative potential and decreased markers of autophagy as compared to their control counterparts. Conversely, HepG2 cell xenografts stably expressing an OXCT1targeting shRNA displayed autophagy activation and reduced proliferation rates. Moreover, the intraperitoneal administration of $\beta$-hydroxybutyrate accelerated tumor progression in vivo, but only when HepG2 cells overexpressed OXCT1 [4]. Circulating $\beta$-hydroxybutyrate was increased in a cohort of 35 HCC patients

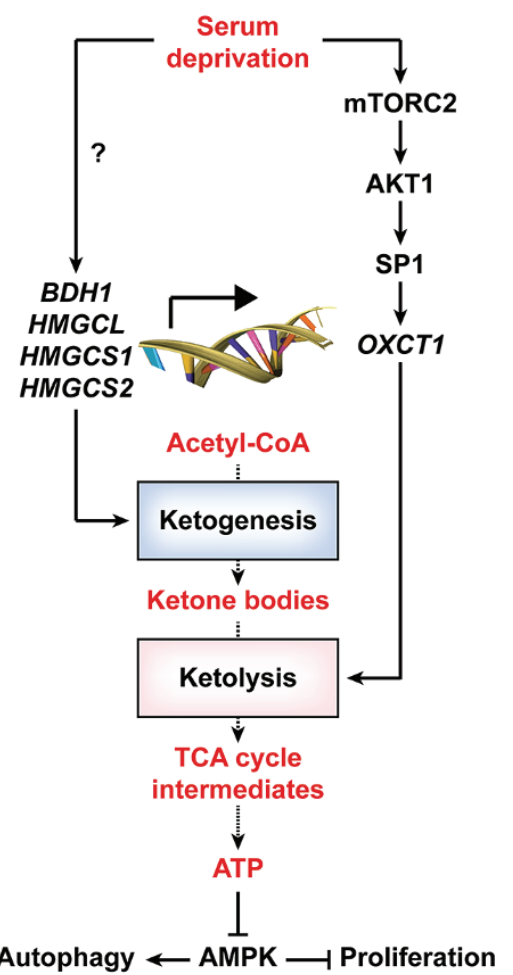

Figure 1 Molecular mechanisms supporting the proliferation of HCC cells despite serum withdrawal. In response to serum deprivation, HCC cells upregulate multiple enzymes involved in ketogenesis, resembling normal hepatocytes. In addition, serumdeprived HCC cells also upregulate OXCT1 expression, via a signal transduction pathway involving the sequential activation of mTORC2, AKT1 and SP1. Thus, contrary to normal hepatocytes, HCC cells also respond to serum deprivation by acquiring the ability to employ endogenous and exogenous ketone bodies for ATP synthesis. Such a capacity allows HCC cells to proliferate despite the nutritional stress imposed by serum withdrawal. TCA, tricyclic acid.

as compared to 29 healthy subjects. Moreover, 20 HCC lesions displayed increased levels of OXCT1 (mRNA), OXCT1 (protein), SP1 (protein) and phosphorylated SP1 (active protein), as compared to adjacent normal tissues. Finally, in a cohort of 148 HCC patients, high expression levels of OXCT1 in malignant cells positively correlated with clinical stage and indicated dismal prognosis in monovariate analysis [4].

In essence, Zhang and co-authors demonstrated that HCC cells acquire the capacity to consume ketone bodies to cope with the nutritional stress imposed by serum starvation, in thus far contrasting with normal hepatocytes, which are only capable of ketogenesis. Moreover, they delineated the signal transduction cascade involving mTORC2, AKT1, SP1 and OXCT1 through which HCC cells activate ketolysis in response to adverse microenvironmental conditions (Figure 1). It will be interesting to determine whether this mechanism can be targeted pharmacologically for the development of new drugs against HCC.

Lorenzo Galluzzi ${ }^{1,2}$, 3, 4, 5, 6
Guido Kroemer
, 3, 4, 5, 7, 8,

${ }^{1}$ Department of Radiation Oncology, Weill Cornell Medical College, New York, NY 10065, USA; ${ }^{2}$ Equipe 11 labellisée Ligue contre le Cancer, Centre de Recherche des Cordeliers, 75006 Paris, France; ${ }^{3}$ INSERM, U1138, 75006 Paris, France, ${ }^{4}$ Université Paris Descartes/Paris V, Sorbonne Paris Cité, 75006 Paris, France; ${ }^{5}$ Université Pierre et Marie Curie/Paris VI, 75006 Paris, France; ${ }^{6}$ Gustave Roussy Comprehensive Cancer Institute, 94805 Villejuif, France; ${ }^{7} \mathrm{Me}-$ tabolomics and Cell Biology Platforms, Gustave Roussy Comprehensive Cancer Institute, 94805 Villejuif, France; ${ }^{8}$ Pôle de Biologie, Hopitâl Européen George Pompidou, AP-HP, 75015 Paris, France; ${ }^{9}$ Department of Women's and Children's Health, Karolinska University Hospital, 17176 Stockholm, Sweden

Correspondence: Lorenzo Galluzzia,

Guido Kroemer ${ }^{\mathrm{b}}$

${ }^{a}$ E-mail: deadoc@vodafone.it

bE-mail: kroemer@orange.fr

\section{References}

1 Hanahan D, Weinberg RA. Cell 2011; 144:646-674.

2 Martinez-Outschoorn UE, Peiris-Pages M, Pestell RG, et al. Nat Rev Clin Oncol 2016 May 4. doi: 10.1038/nrclinonc.2016.60

3 Galluzzi L, Kepp O, Vander Heiden MG, et al. Nat Rev Drug Discov 2013; 12:829-846.

4 Huang D, Li T, Wang L, et al. Cell Res 2016; 26:1112-1130.

$5 \quad$ Huang D, Li T, Li X, et al. Cell Rep 2014; 8:1930-1942.

6 Pietrocola F, Galluzzi L, Bravo-San Pedro JM, et al. Cell Metab 2015; 21:805-821.

7 Llovet JM, Zucman-Rossi J, Pikarsky E, et al. Nat Rev Dis Primers 2016; 2:16018.

8 Shimobayashi M, Hall MN. Nat Rev Mol Cell Biol 2014; 15:155-162.

9 Hardie DG, Ross FA, Hawley SA. Nat Rev Mol Cell Biol 2012; 13:251-262.

10 Galluzzi L, Pietrocola F, Bravo-San Pedro $\mathrm{JM}$, et al. EMBO J 2015; 34:856-880.

11 Sica V, Galluzzi L, Bravo-San Pedro JM, et al. Mol Cell 2015; 59:522-539. 